\title{
Hypothermia with Pneumonia: A Rare Presentation of Brucellosis
}

\author{
Hossam El Ghoussein Mohamed Osama Hegazi \\ Department of Medicine, Al Adan Hospital, Hadiya, Kuwait
}

\section{Key Words}

Brucellosis $\cdot$ Hypothermia $\cdot$ Pneumonia

\begin{abstract}
Objective: To report a very rare form of brucellosis presenting with hypothermia and pneumonia. Clinical Presentation and Intervention: A 41-year-old male shepherd presented with a depressed level of consciousness. Clinically, his rectal temperature was $29.5^{\circ} \mathrm{C}$, and he was cold, apathetic, hyporeflexic, and hypotensive, with atrial fibrillation. He had clinical and radiological evidence of bilateral bronchopneumonia. Blood culture and serologic testing were positive for Brucella melitensis. The patient recovered completely after proper management of the hypothermia and treatment of the brucellosis with antibiotics (doxycycline $100 \mathrm{mg}$ orally twice daily for 6 weeks and streptomycin $1 \mathrm{~g}$ i.m. daily for 21 days). Conclusion: This case shows that brucellosis should be considered in the differential diagnosis of septicemic patients presenting with hypothermia.
\end{abstract}

Copyright ๑ 2011 S. Karger AG, Basel

\section{Introduction}

Brucellosis is a bacterial zoonotic disease that is found worldwide. Although it is well controlled in most developed countries, it is still common in the Middle East, Asia, Africa, South and Central America, the Mediterranean basin, and the Caribbean $[1,2]$. Brucellosis usually presents with fever and constitutional symptoms. Physical examination is generally nonspecific, though lymphadenopathy, hepatomegaly, or splenomegaly is often present [1]. Focal manifestations of the disease may involve the musculoskeletal, genitourinary, gastrointestinal, nervous, or cardiovascular systems [1]. Respiratory complications of brucellosis are considered rare [1]. Hypothermia has not been reported to be associated with brucellosis, except in one case report [3]. We describe a case of brucellosis that presented with hypothermia and pneumonia.

\section{Case Report}

A 41-year-old male shepherd presented with an acute state of confusion. He had a history of fever, malaise, dry cough, and repeated vomiting for the last 4 days. There was no history of alcohol ingestion, drug intake, trauma, or cold exposure. Clinical examination showed cold skin, apathy and hyporeflexia with no focal neurological deficits. The rectal temperature was $29.5^{\circ} \mathrm{C}$. He had an irregular pulse with a heart rate of 56 beats/min and a blood pressure of $95 / 60 \mathrm{~mm} \mathrm{Hg}$. He was neither pale nor jaundiced, and did not show lymphadenopathy. His chest showed bilateral scattered crepitations, and examination of the heart and abdomen was unremarkable. Chest X-ray showed bilateral mid-zonal consolidations with an air bronchogram, suggesting bronchopneumonic patches. Electrocardiogram (ECG) showed atrial fibrillation, prolonged Q-T interval, and J (Osborn) waves (fig. 1). Blood investigations revealed: WBC: $5.5 \times 10^{9} /$ l; neutrophils: $2.5 \times 10^{9} / 1$; lymphocytes: $2.4 \times 10^{9} / 1$; hemoglobin: $12.2 \mathrm{~g} / \mathrm{dl}$; mean cardiac volume: $81 \mathrm{fl}$; platelets: $167 \times 10^{9} / \mathrm{l}$; bilirubin: $26 \mu \mathrm{mol} / \mathrm{l}$; aspartate aminotransferase: 192 IU/l (normal 15-40); alanine aminotrans-

\section{KARGER}

Fax +4161306 1234

E-Mail karger@karger.ch

www.karger.com
(C) 2011 S. Karger AG, Basel

1011-7571/11/0205-0485\$38.00/0

Accessible online at:

www.karger.com/mpp
Dr. Mohamed Osama Hegazi

Department of Medicine, Al Adan Hospital

PO Box 262

Hadiya 52853 (Kuwait)

Tel. +9659740 3085, E-Mail drosama02@gmail.com 
Fig. 1. ECG of the patient showing slow atrial fibrillation, prolonged Q-T interval, and J wave (arrows).

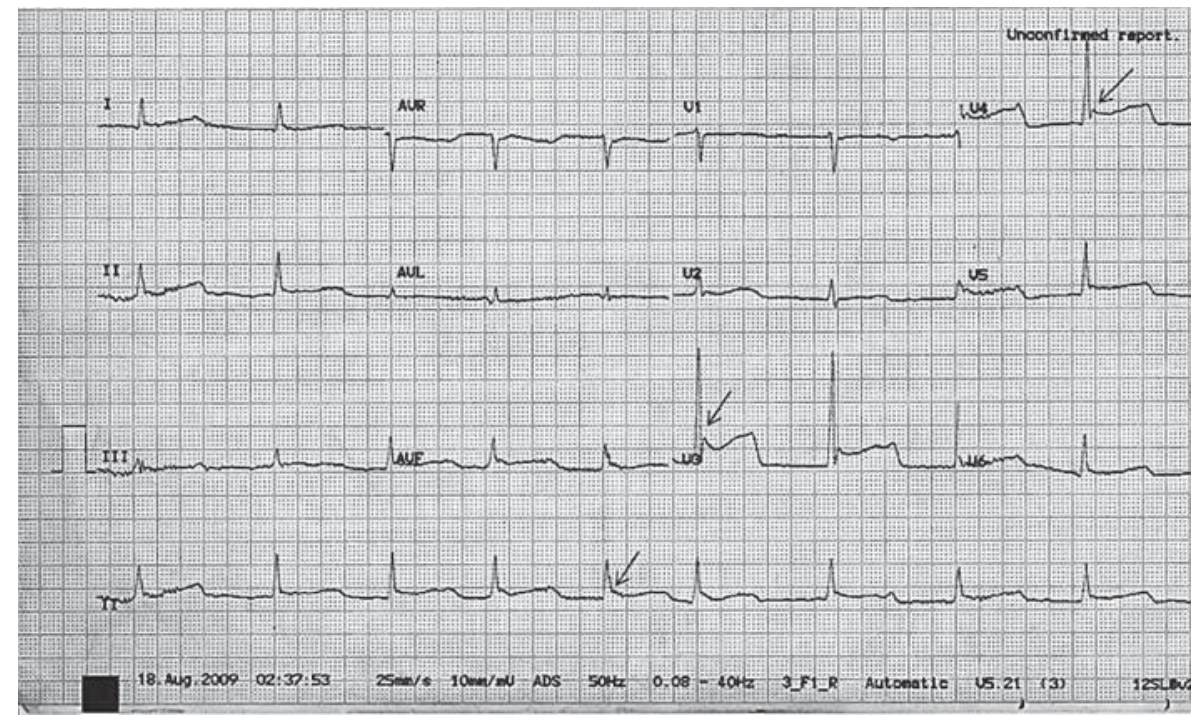

ferase: $160 \mathrm{U} / 1$ (normal 0-37); alkaline phosphatase: $232 \mathrm{U} / \mathrm{l}$ (normal 40-129); lactic acid: $6.6 \mathrm{mmol} / \mathrm{l}$ (normal 0.5-1.5). Arterial blood gas analysis showed: $\mathrm{pH}$ : 7.23; $\mathrm{PO}_{2}: 7 \mathrm{KPa} ; \mathrm{PCO}_{2}: 4 \mathrm{KPa}$; bicarbonate: $20 \mathrm{mmol} / \mathrm{l}$. Serum urea, creatinine, electrolytes, sugar, calcium, magnesium, phosphate, as well as thyroid and adrenal function tests were normal. Echocardiography was normal and abdominal ultrasonography showed splenomegaly with normal sonographic appearance of the liver. Cerebrospinal fluid cell count and biochemical analysis were normal. A preliminary diagnosis of sepsis with hypothermia was made. The patient was managed with oxygen, cardiac monitoring, active and passive external re-warming, warm i.v. fluids and i.v. piperacillin/tazobactam (4.5 g every $8 \mathrm{~h}$ ). The patient's sensorium and hemodynamic state improved, and he started to develop fever. ECG showed a return to sinus rhythm, normalization of the Q-T interval, and disappearance of the Osborn wave. A positive blood culture for Brucella after 5 days of incubation and a 1/320 titer of Brucella standard agglutination test were obtained. The blood culture showed characteristics indicating Brucella melitensis $\left(\mathrm{H}_{2} \mathrm{~S}\right.$ production negative, Tiblisi phage lysis negative, Weybridge phage lysis negative, etc.). Treatment in the form of doxycycline $100 \mathrm{mg}$ orally twice daily for 6 weeks plus streptomycin $1 \mathrm{~g}$ i.m. daily for the first 21 days was given, and the patient completely recovered with full clinical improvement, clearance of chest X-ray consolidations, and a return of hepatic transaminase levels to normal values.

\section{Discussion}

Brucellosis is a zoonotic febrile illness caused by infection with the Gram-negative intracellular coccobacilli Brucella species. Transmission of brucellosis to humans occurs through the consumption of infected unpasteurized animal milk products, through direct contact with infected animal parts (by inoculation through cuts or abrasions of the skin and mucous membranes), and through the inhalation of infected aerosolized particles [1]. Brucellosis is an occupational disease in shepherds, abattoir workers, veterinarians, dairy industry professionals, and personnel in microbiologic laboratories [1]. Four species of Brucella can cause human disease: B. melitensis, B. abortus, B. suis, and B. canis [1]. Infection with $B$. melitensis accounts for the vast majority of cases worldwide as well as in Kuwait $[1,4]$. Clinical disease is common in the Middle East and Asia [2]. Fever, chills, sweats, gastrointestinal symptoms, headache, and musculoskeletal symptoms are the usual presenting features, with the musculoskeletal, genitourinary, and nervous systems being the most common sites of focal infection and complications $[5,6]$. Fever is a presenting symptom in $91 \%$ of cases, and it is described as an invariable feature of brucellosis $[1,5]$.

Hypothermia is a life-threatening condition that is defined as a core temperature below $35^{\circ} \mathrm{C}$. Our patient had a moderate degree of hypothermia as his rectal temperature was $29.5^{\circ} \mathrm{C}$. ECG changes in hypothermia include prolongation of all ECG intervals (PR, RR, QRS, and QT), J (Osborn) waves, sinus bradycardia, atrial fibrillation, and junctional bradycardia [7]. The changes in our patient's ECG were reversible after correction of the hypothermia. Severe hypothermia can lead to pulmonary edema, oliguria, areflexia, coma, hypotension, bradycardia, ventricular arrhythmias (including ventricular fibrillation), and asystole [8]. Management should be guided by the cause, degree of hypothermia and coexisting conditions. Initially, patients should be re-warmed, while look- 
ing for a potential cause. Cardiac monitoring is essential and endotracheal intubation should be considered. Volume resuscitation with crystalloid solutions is indicated and dopamine should be considered in refractory cases. Even with modern supportive care, the in-hospital mortality of patients with moderate or severe accidental hypothermia approaches $40 \%$ [9]. Fortunately, initial management with re-warming, oxygen therapy, cardiac monitoring, i.v. fluids, and antibiotics were sufficient for our patient to survive.

In spite of the fact that hypothermia can be induced by sepsis, especially with a Gram-negative organism, brucellosis is very rarely described with hypothermia. To our knowledge and after an extensive search, only one case of brucellosis presenting with hypothermia has been published as a case report (in a letter to the editor) by Casariego Vales et al. [3]. Unlike our patient, the published case was of an elderly 78-year-old man. Both the previously published case and our case did not have a history of cold exposure, had positive blood cultures for Brucella melitensis, and recovered after therapy.

Involvement of the respiratory system in brucellosis is an acknowledged but rare event that is only occasionally described in literature. Pappas et al. [10] described 37 cases of brucellosis with respiratory complications in the form of pneumonia, bronchopneumonia, pleural effusion, and paroxysmal dry cough. Al-Anazi et al. [11] described a case of hemorrhagic pleural effusion due to brucellosis. In a study from Saudi Arabia, pneumonia was a clinical feature of brucellosis in $1.3 \%$ of cases [12].

\section{Conclusion}

This case showed that brucellosis should be considered in the differential diagnosis of septicemic patients presenting with hypothermia.

\section{References}

1 Pappas G, Akritidis N, Bosilkovski M, Tsianos E: Brucellosis. N Engl J Med 2005;352: 2325-2336.

-2 Dimitrov T, Panigrahi D, Emara M, Awni F, Passadilla R: Seroepidemiological and microbiological study of brucellosis in Kuwait. Med Princ Pract 2004;13:215-219.

$\checkmark 3$ Casariego Vales E, Lopez Alvarez MJ, Varela Otero J, Corredoira Sanchez JC: Hypothermia as a presenting sign of brucellosis. Clin Infect Dis 1993;16:588.

$\checkmark 4$ Al-Nakkas A, Mustafa AS, Wright SG: Large-scale evaluation of a single-tube nested PCR for the laboratory diagnosis of human brucellosis in Kuwait. J Med Microbiol 2005;54:727-730.
5 Mousa AR, Elhag KM, Khogali M, Marafie AA: The nature of human brucellosis in $\mathrm{Ku}$ wait: study of 379 cases. Rev Infect Dis 1988; 10:211-217.

6 Lulu AR, Araj GF, Khateeb MI, Mustafa MY, Yusuf AR, Fenech FF: Human brucellosis in Kuwait: a prospective study of 400 cases. Q J Med 1988;66:39-54.

7 de Souza D, Riera AR, Bombig MT, Francisco YA, Brollo L, Filho BL, Dubner S, Schapachnik E, Povoa R: Electrocardiographic changes by accidental hypothermia in an urban and a tropical region. J Electrocardiol 2007;40:47-52.

8 Jolly BT, Ghezzi KT: Accidental hypothermia. Emerg Med Clin North Am 1992;10: 311.
9 Vassal T, Benoit-Gonin B, Carrat F, Guidet B, Maury E, Offenstadt G: Severe accidental hypothermia treated in an ICU: prognosis and outcome. Chest 2001;120:1998-2003.

10 Pappas G, Bosilkovski M, Akritidis N, Mastora M, Krteva L, Tsianos E: Brucellosis and the respiratory system. Clin Infect Dis 2003; 37:95-99.

11 Al-Anazi AR, Aziz S, Fouda MA: Brucellosis: haemorrhagic pleural effusion. Med Princ Pract 2005;14:118-120.

12 Fallatah SM, Oduloju AJ, Al-Dusari SN, Fakunle YM: Human brucellosis in Northern Saudi Arabia. Saudi Med J 2005;26:15621566. 